\title{
Development of error reduction model using Bayesian filter for AUV navigating under moving ice
}

\author{
Jimin Hwang \\ National Centre for Maritime \\ Engineering \& Hydrodynamics \\ Australian Maritime College, \\ University of Tasmania \\ Launceston, Australia \\ jimin.hwang@utas.edu.au
}

\author{
Shuangshuang Fan \\ National Centre for Maritime \\ Engineering \& Hydrodynamics \\ Australian Maritime College, \\ University of Tasmania \\ Launceston, Australia \\ shuangshuang.fan@utas.edu.au
}

\author{
Peter King \\ National Centre for Maritime \\ Engineering \& Hydrodynamics \\ Australian Maritime College, \\ University of Tasmania \\ Launceston, Australia \\ p.d.king@utas.edu.au
}

\author{
Alexander Forrest \\ Department of Civil and \\ Environmental Engineering \\ University of California \\ Davis, USA \\ alforrest@ucdavis.edu
}

\begin{abstract}
Doppler Velocity Logs (DVL) can provide a simple under water navigation aid for Autonomous Underwater Vehicles (AUV) by measuring relative velocities with respect to the speed over ground. A valid reference velocity is difficult to calculate when this approach is applied under a moving frame of reference such as drifting ice. The primary challenge of under-ice localization is to accurately estimate the AUV location and its trajectory in the global coordinate system when DVL measurements are being made relative to a constantly drifting ice surface. In this paper, the author introduces and compares two types of error sources, scale factor error of DVL and navigation error due to ice drift. An error reduction model using a Bayesian filter algorithm is developed for improved estimations, in conjunction with a novel correction method for accurate AUV navigation under ice. The concept of shift factor is introduced in this paper as the key to solve both error sources. Having the knowledge of the true beacon location, shift factors in vector quantity are extracted based on the collected relative velocity profiles by DVL. The shift factors are directly applied to update the final AUV location. The result presents approximately $\mathbf{7 0 . 8 \%}$ of maximum error reduction. The impact of survey pattern, bearing angle to the beacon, pinging frequency on the accuracy of the vehicle localisation are discussed.
\end{abstract}

Keywords-Autonomous underwater vehicles (AUVs), underice AUV navigation, Doppler Velocity Logs (DVLs), acoustic positioning system, a single beacon, moving frame of reference, Bayesian filter algorithm, shift factors

\section{INTRODUCTION}

The evolution of technology for the last decade has led to revolutionary improvements in the development of Autonomous Underwater Vehicles (AUV) enabling them to access places where manned or tethered vehicles physically cannot or are logistically prohibitive to reach; such as deep ocean floor, hazardous areas and under ice, particularly in Polar Regions. Increasing academic and industrial interests of the Polar Regions have triggered demand for effective navigation during under-ice scientific missions [1], [2].

Speed over ground of the vehicles used for those missions is measured often using Doppler Velocity Logs (DVL). Reference [3] concisely explained how DVL works; sending out the oscillating acoustic signals and sampling the returned signals. DVL has proven to be a popular, inexpensive, realtime accomplishable technique in dead reckoning despite the time-dependent accumulated errors.
Generally, DVL has a bottom-lock range of 30 to $200 \mathrm{~m}$ latitude above the seafloor with 300 to $1200 \mathrm{kHz}$ of frequency range [4]. Increasing resolution requires higher frequency. Since the DVL is designed to provide relative velocity to a fixed reference object, it becomes significantly less accurate when the reference object is moving. Error rate due to ice movement could add serious offset to the DVL's standard deviation [5]. During actual field operations, the cumulative error possibly translates into high position uncertainty of bathymetry, hence results in failure of homing attempts. This paper will analyse the navigation problems when AUV is operating under moving ice by simulating the various scenarios including basic DVL cases and a pair of a beacon and a transducer with Bayesian filter using MATLAB. Description of each scenario will be detailed in the methodology section. The results of the proposed correction method will be presented and discussed in result and discussion section, respectively.

\section{Methodology}

The testing scenarios has the same general arrangement, but different ice motion variables as depicted in Fig. 1. A beacon can be installed on the surface vessel (side or bottom of the hull mounted) or the bottom of the ice. It regularly sends pings to the transducer on AUV as it navigates under the ice. Subsequently the error reduction algorithm using Bayesian filter analyses the locational data and generate an improved estimation. The improvement in error reduction rates is to be numerically quantified.

\section{A. Problem modelling}

Two simple scenarios will be simulated to show the extent of issues when navigating with DVL. They are labelled according to the characteristic of the simulation. In $D D-1$, the relative velocity is measured between AUV and earth frame of reference (EFR), such as when the DVL is tracking the seafloor. In $D D-2$, relative velocity is measured between AUV

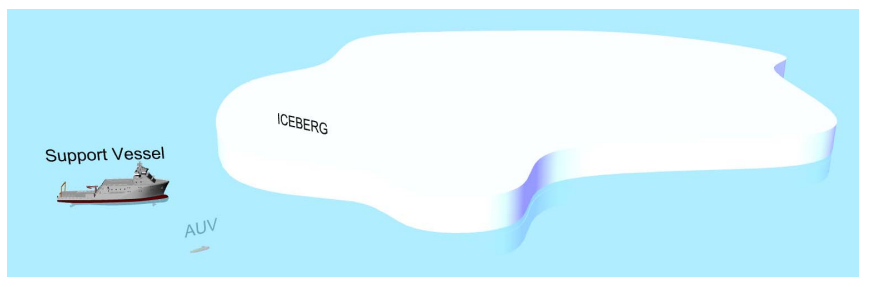

Fig. 1. General arrangement of support vessel and AUV operation under ice. 
and the ice frame of reference (IFR), such as when the DVL is tracking the underside of moving ice. $D D$ stands for DeadReckoning using DVL.

\section{B. Mathematical model}

Mathematical models were successively developed to simulate velocity measured by DVL and the motion of AUV in both global (earth) and local (ice) coordinate system so that the collected coordinate data are interchangeable. Firstly, AUV velocity profiles are derived in the local coordinate system, based on three components of DVL velocity measurements: heading angle, forward and lateral speed. The velocity components of the DVL then converted to East and North velocity components respectively as illustrated Fig. 2. The multiplication matrix for velocity in a fixed frame of reference is presented in (1).

$$
\left[\begin{array}{c}
V_{E} \\
V_{N}
\end{array}\right]=\left[\begin{array}{cc}
\cos \theta & \sin \theta \\
-\sin \theta & \cos \theta
\end{array}\right]\left[\begin{array}{c}
V_{\text {Lat }} \\
V_{F w d}
\end{array}\right]
$$

Position coordinates $(x, y)$ in (2) and (3) are obtained by integrating the instantaneous velocity equation, $v=d x / d t$.

$$
\begin{aligned}
& x_{t}=x_{t-1}+\left(\frac{V_{E_{t}}-V_{E_{t-1}}}{2}\right) d t \\
& y_{t}=y_{t-1}+\left(\frac{V_{N_{t}}-V_{N_{t-1}}}{2}\right) d t
\end{aligned}
$$

The final modified equation describes three components comprised of total velocity vectors to define absolute AUV position in a moving frame of reference as shown in (4). Owing to the rotation of the reference frame $\delta$, true heading angle changes from $\theta$ degree(s) to $(\theta+\delta)$ degree(s).

$$
\left[\begin{array}{c}
V_{E_{T+R}} \\
V_{N_{T+R}}
\end{array}\right]=\left[\begin{array}{cc}
\cos (\delta+\theta) & \sin (\delta+\theta) \\
-\sin (\delta+\theta) & \cos (\delta+\theta)
\end{array}\right]\left[\begin{array}{c}
V_{L a t} \\
V_{F w d}
\end{array}\right]+\left[\begin{array}{c}
V_{E_{\text {ice }}} \\
V_{N_{\text {ice }}}
\end{array}\right]+\left[\begin{array}{c}
r \omega \cdot \sin \varphi-\delta \\
-r \omega \cdot \cos \varphi-\delta
\end{array}\right]
$$

It is subsequently modified to take into account the moving frame of reference as shown in (5) and (6).

$$
\begin{gathered}
\text { East }: x_{t}=x_{t-1}+\left(\frac{V_{E_{T+R_{t}}}-V_{E_{T+R_{t-1}}}}{2}\right) d t \\
\text { North }: y_{t}=y_{t-1}+\left(\frac{V_{N_{T+R_{t}}}-V_{N_{T+R_{t-1}}}}{2}\right) d t
\end{gathered}
$$

\section{Beacon localisation}

Acoustic positioning using beacon(s) is a common method used to aid AUV underwater navigation by using trilateral or multilateral localisation. It calculates the location of the target

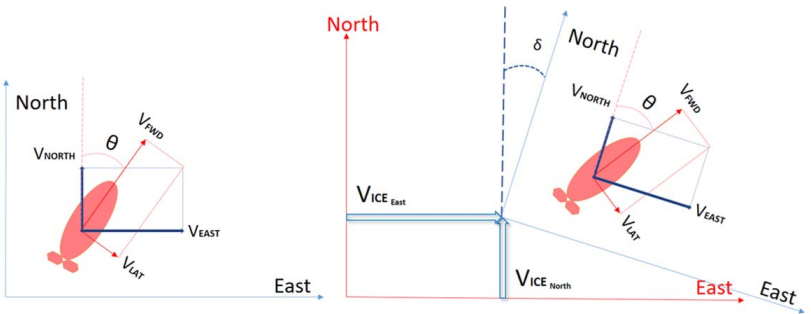

Fig. 2. AUV velocity vector components and heading angle of the two models being converted in local coordinate system (left) and global coordinate system (right) respectively. object by measuring distance, using the geometry of a series of circles (2-dimensional) or spheres (3-dimensional). Ideally, a minimum of four beacons are installed at work site to improve positioning accuracy and to provide flexibility to survey a large area [6]. Single or multiple beacon(s) localisation has been widely used for navigation aid by a number of researchers such as [7]-[9]. The accuracy of the acoustic positioning is generally dependent on the frequency of ping to be received, the number of referencing points, and the direction of the moving target. Whilst the target object constantly moves, the beacon also continuously pings an acoustic signal.

When only one beacon is available, multiple sets of acoustic return signals from the moving body are used to find the beacon by searching for intersected point as shown in Fig. 3. Reference [10] utilised a single beacon for AUV localisation and homing using the trilateration technique.

\section{Bayesian filtering}

Once an AUV is underwater, it has to be able to localise itself based on the in-situ information received from the sensors. Localisation, in this context, refers to estimation of its position in global coordinates. The estimation, also referred to as belief, reflects internal knowledge of position of the AUV with respect to the surroundings [11]. The belief provides the probability of the AUV at a particular location with the history of sensor measurements for all possible locations.

Recursive Bayesian estimation, also called Bayes filter algorithm is one of the most general probabilistic approaches to calculate beliefs. The unknown probability density function (PDF) estimates recursively over time based on measurements and mathematical process model. Originally introduced in 1763 by Bayes [13], the Bayesian estimation (or Bayesian theorem) was genetic simple mathematical probabilistic model for location estimation as presented in (7):

$$
P(H \mid D)_{\text {Posterior }}=\frac{P(H)_{\text {prior }} \cdot P(D \mid H)_{\text {Likelihood }}}{P(D)_{\text {NormalisationFactor }}}
$$

Fundamentally, the Bayesian filter consists of two essential steps [12]: a transition update (prediction) and sensor update (measurement). A belief of the state, $x_{t}$ in the first transition step is calculated based on the prior belief of the state, $x_{t-1}$ by integrating the product of the prior assigned to the state, $x_{t-1}$ and the probability of $u_{t}$ inducing of the measurement, $z_{t}$ allowed to be observed, then divided by normalisation constant. Those two prediction and measurement steps are mathematically

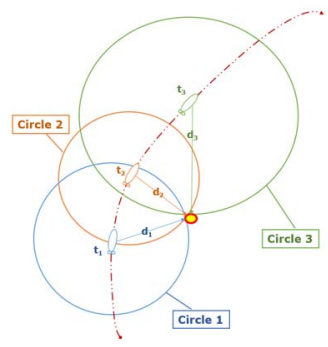

Fig. 3. An illustration showing the ordinary localization method using the returned pings from a single beacon. While $t$ stands for time, $d$ stands for distance, three coloured circles refer to the ranges of acoustic signal, and yellow dot is the location of the beacon. 
expressed respectively as follows.

$$
\begin{gathered}
\text { prediction }=\int P\left(x_{t} \mid x_{t-1}, u_{t}\right) \cdot x_{t-1} d x \\
\text { measurement }=\eta\left(P\left(x_{t} \mid x_{t-1}, u_{t}\right) \cdot \text { prediction }\right)
\end{gathered}
$$

This type of grid-based approach tessellates the given space into small patches. Each grid cell contains the belief that the target is in each cell. The summation of beliefs roughly answers the question of the probability that the target is at location of $x$, when this history of sensor measurements $z_{t}$, for all possible locations $x_{t}$. The main advantage of this approach is that each reading can represent arbitrary distributions over the discrete state space [13]. The metric approximations can also provide accurate location estimates with high robustness to sensor noise [14]. Unlike the beacon localisation using general trilateration, a single beacon can be utilised without losing much accuracy when the beacon resource is limited.

\section{E. Simulation scenarios description}

Two scenarios were set up with the same operating environment involving an AUV with DVL, and a support vessel next to the ice. The first case was with the beacon mounted on the bottom of the ice while the second one was on the supporting vessel. These two scenarios were labelled as Bayesian with Beacon $(B B)-1$ and 2 as illustrate in Fig. 4. Since the beacon was moving with the ice in $B B-1$, the estimated coordinates were in local coordinate system. All navigational information including DVL data refers to the ice. Therefore, the only relevant frame of reference was the moving ice. In this simulation, global coordinates are untraceable unless the precise ice-movement information is known. In $B B$ 2 , the beacon was at the fixed position in global coordinate system, whilst DVL remained relative to the ice. As a result, two relevant coordinate systems are involved, local for the DVL and global for the beacon.

\section{F. Beacon localisation}

The global coordinate of the beacon is known from the beginning in the $B B-2$ and remained at the fixed location. At the end of every simulation, the difference between the AUV position estimate and true beacon position could be a shift

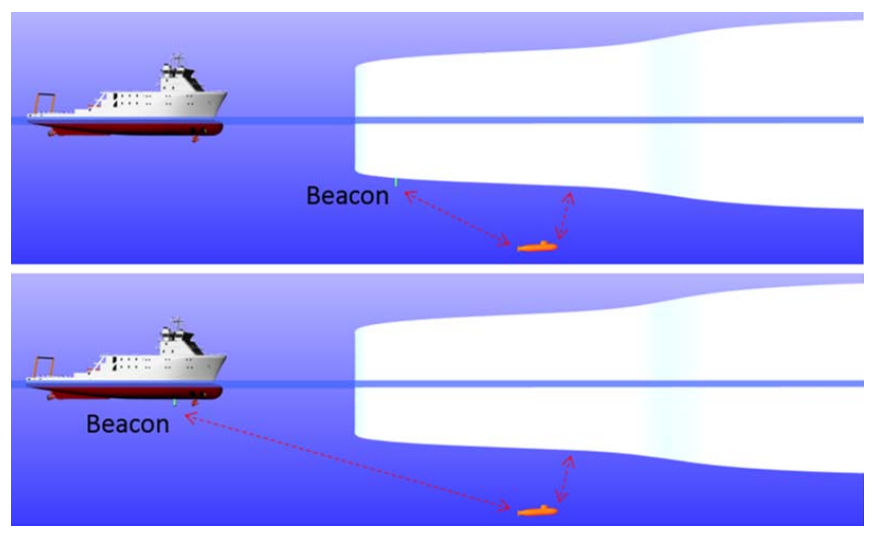

Fig. 4. A schematic drawing the simulation scenario $B B-1$. The dashed arrows indicate the communication between the beacon and the transducer on AUV. (Top) A schematic drawing the simulation scenario $B B-2$. The dashed arrows indicate the communication between the beacon and the transducer on AUV. (Bottom) factor to correct the estimated position of the AUV as shown in Fig. 5. A simple mathematical expression used in the algorithm is shown in (10). Finally, equation (11) and (12) were used throughout the analysis of all simulated results.

$$
\begin{gathered}
A U V\left(x_{\text {update }}, y_{\text {update }}\right)=\operatorname{AUV}\left(x_{\text {estimate }}, y_{\text {estimate }}\right)+\operatorname{Beacon}\left(x_{\text {error }}, y_{\text {error }}\right) \\
\text { Displacement }[\mathrm{m}]=\sqrt{\left(x_{\text {estimate }}-x_{\text {true }}\right)^{2}+\left(y_{\text {estimate }}-y_{\text {true }}\right)^{2}} \\
\text { error rate }[\%]=\frac{\text { Diaplacement }[\mathrm{m}]}{\text { total travel distance }[\mathrm{m}]}
\end{gathered}
$$

\section{Simulation Results}

The results are presented in order of dead-reckoning simulations ( $D D-1$ and $D D-2)$ and Bayesian simulations $(B B-1$ and $B B-2)$. In addition, the beacon pinging interval convergence study was conducted.

\section{A. DD-1: Navigation accuracy of DVL}

As a preliminary investigation, a basic AUV deadreckoning navigation with DVL data was simulated in both EFR and IFR. Actual data set from the previous operation of the Memorial University of Newfoundland's Explorer AUV in 2015 was utilized. The calculated positions were then compared with its surface global positioning system (GPS) data. Total travelled distance of the AUV was $1.73 \mathrm{~km}$ for 1,000 seconds. AUV trajectory is presented in Fig. 6. The red line indicates the estimated AUV position based on DVL data, while the blue line is defined by GPS. The unit of the received data was in geodetic coordinate but converted to Universal Transverse Mercator (UTM).

The error was approximately $2.87 \%$ with $50 \mathrm{~m}$ difference at the final destination. It was observed that error built over time when relying on dead reckoning solely. Statistics of the first simulation is tabulated below in Table I.

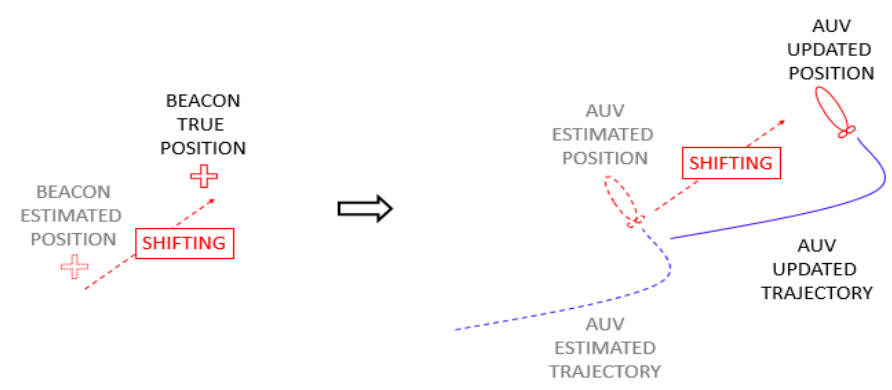

Fig. 5. Correction method to update the final AUV position using the shift factors extracted from the beacon positional errors.

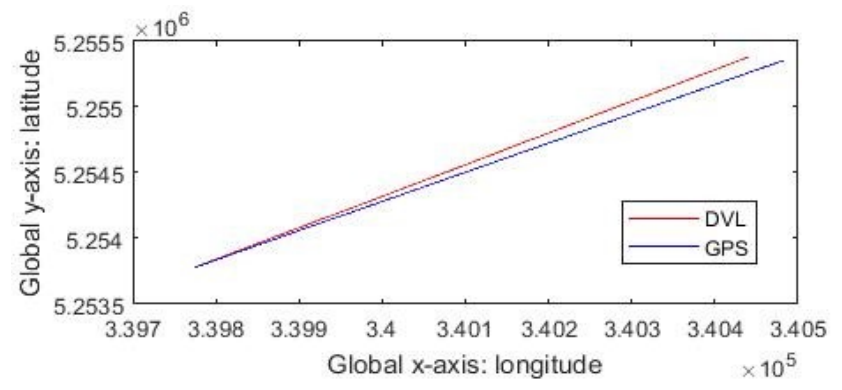

Fig. 6. A plot showing the results of the simulation $D D-1$. 
Table I. OPERATING CONDITIONS AND RESULTS OF SIMULATION $D D-1$.

\begin{tabular}{|c|c|}
\hline Particular & Value \\
\hline Travelled Distance $(\mathrm{km})$ & 1.73 \\
\hline Operating time $(\mathrm{s})$ & 1000 \\
\hline Average Velocity $(\mathrm{m} / \mathrm{s})$ & 1.6 \\
\hline Error in total distance $(\mathrm{m})$ & 49.7 \\
\hline Error rate $(\%)$ & 2.87 \\
\hline
\end{tabular}

\section{B. DD-2: Navigation error due to ice-drift}

Common DVL data sets were generated to recreate more realistic problems of under ice AUV operation. Based on typical iceberg translational speeds of $0.03-0.08 \mathrm{~m} / \mathrm{s}$ and rotational rates of $5^{\circ}-10^{\circ}$ per hour [5], three scenarios were run varying translation speeds and rotation angles. Only the errors by ice drift were in effect. AUV trajectory is plotted in Fig. 7. The red circles indicate the estimates defined by DVL data, whilst the blue lines were the true trajectory of AUV. Having the various ice transitional velocities could drastically alter the overall localisation outcomes.

Constantly translating and rotating ice drift caused much confusion as a subtle increase of the ice translating speeds substantially increases error rates. The error due to ice-drift was greater than the navigation error from observation. Table II summarises details of the simulation $D D-2$.

\section{BB-1: Beacon mounted on the bottom of the moving ice}

In $B B-1$, various pattern test and beacon location independence study were investigated. Only navigation errors were assumed to exist in $B B-1$. Simulation set-up was similar to the normal seafloor navigation except the entire reference frame is translating and rotating. Three AUV operating patterns were tested: the straight line, the right-angle lawn mower, and the spiral shape. The beacon pinging interval was once per 120 seconds. The total error was reduced when more complicated
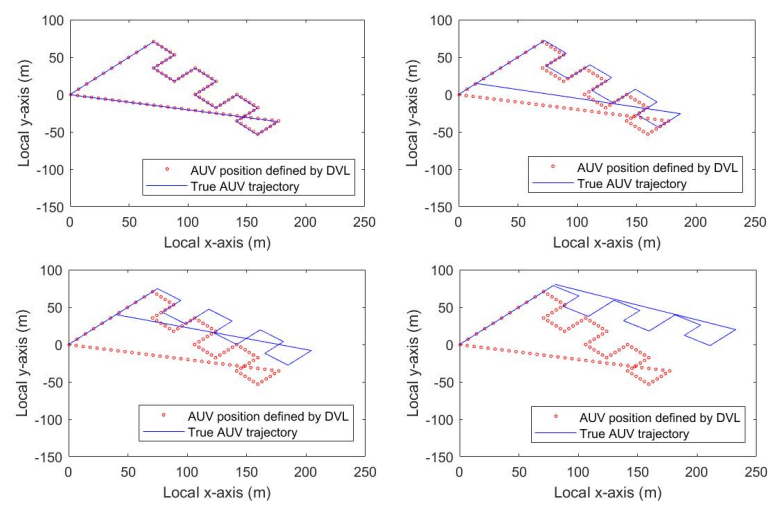

Fig. 7. Plots showing the results of Simulation $D D-2$ series (top left: $D D-2-0$, top right: $D D-2-1$, bottom left: $D D-2-2$, bottom right: $D D-2-3$ )

Table II. OPERATING CONDITIONS AND RESULTS OF SIMULATION $D D-2$.

\begin{tabular}{|c|c|c|c|c|}
\hline Particulars & DD-2-0 & DD-2-1 & DD-2-2 & DD-2-3 \\
\hline Travelled distance (m) & \multicolumn{4}{|c|}{1160} \\
\hline Vehicle velocity (m/s) & \multicolumn{5}{|c|}{$1.0-2.0$} \\
\hline Ice velocity to East (m/s) & 0 & 0.03 & 0.08 & 0.16 \\
\hline Ice velocity to North (m/s) & 0 & 0.03 & 0.08 & 0.16 \\
\hline Ice angular velocity (\%/h) & 0 & 5 & 8 & 10 \\
\hline Total displacement (m) & 0 & 21.1 & 56.5 & 113.5 \\
\hline $\begin{array}{c}\text { Error rate with respect to } \\
\text { total travelled distance (\%) }\end{array}$ & 0 & 1.82 & 4.87 & 9.79 \\
\hline
\end{tabular}

pattern with various bearing angles was performed.

\section{1) BB-1-1: Straight-line simulation results}

An arbitrary rectangular operating area under the ice was set $1000 \mathrm{~m}$ by $1000 \mathrm{~m}$ with the origin placed at $(0,0)$. The beacon was placed at $(900,900)$ which gives $45^{\circ}$ bearing angle when the AUV is heading to the North. From the origin point, AUV follows a straight line with various bearing angles from zero to $360^{\circ}$. Simulation was repeated 100 times for each angle. DVL bias error and scale factor error was assumed to be $0.1^{\circ}$ and $0.01 \%$ respectively. Fig. 8 displays how bearing angle influenced the overall localisation error rates. It was observed that error rates peaked when bearing angle was either $0^{\circ}$ or $180^{\circ}$ which corresponded with heading angle of $45^{\circ}$ and $225^{\circ}$ to North, respectively. When heading directly toward or away from the beacon, localisation accuracy decreased. The maximum error occurred at bearing angle of $174^{\circ}$ and $184^{\circ}$, both showing $13.28 \%$ error rates. Error rate of $11.74 \%$ was observed at bearing angle of $0^{\circ}$. Overall error rates were ranged between minimum $0.2 \%$ and maximum $13.28 \%$.

While the magnitude of the maximum error at both angles were similar, gradient of error was different: at bearing angle of $0^{\circ}$ it was a lot steeper compared with $180^{\circ}$, meaning that error control could be more robust when AUV is directly heading toward the beacon than the opposite.

\section{2) BB-1-2 and BB-1-3: Lawn-mower and Spiral results}

Two common operating patterns, lawn-mower pattern $(B B$ 1-2) and spiral shape (BB-1-3) were simulated under similar conditions. Each run was repeated 100 times with the beacon being placed at a random position to ensure that the results were independent of the beacon location. The visual patterns are shown in Fig. 9. Total travelled distance of $B B-1-2$ and $B B-$ $1-3$ was $3.1 \mathrm{~km}$ and $5.0 \mathrm{~km}$ respectively. Small circles represent randomly located beacon position by algorithm throughout the repeated simulations. The red lines indicate the estimated path based on DVL data, while the blue lines were the actual AUV

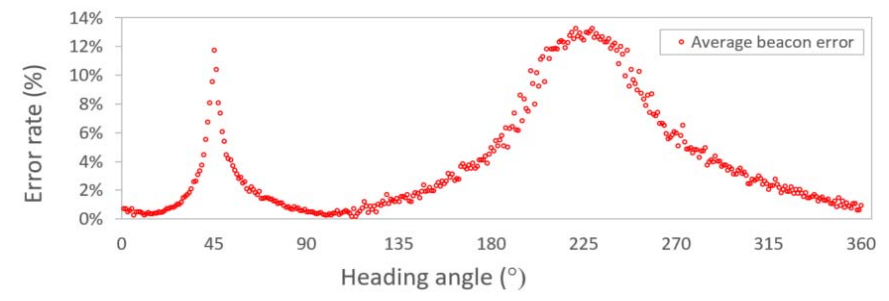

Fig. 8. Averaged error rates of acoustic localization against heading angles from $B B-1-1$ straight-line simulation results
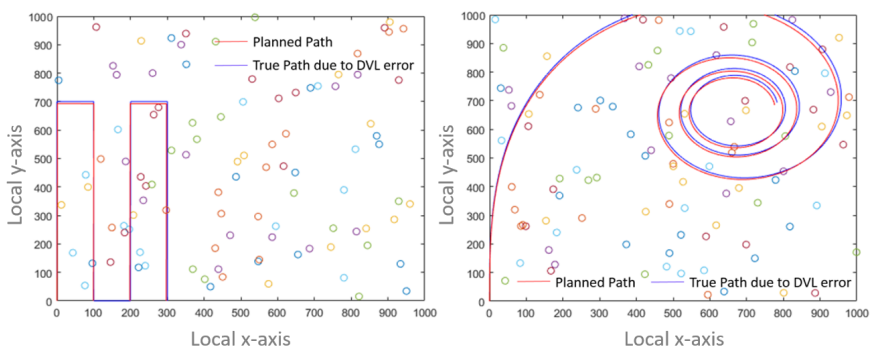

Fig. 9. $B B-1-2$ lawn mower pattern (left) and $B B-1-3$ spiral simulation (right) with coloured marked circles being randomly assigned beacon positions. 
path during operation. Difference between those two trajectories were assumed due to DVL navigation error.

The average error rates were $0.14 \%(B B-1-2)$ and $0.29 \%$ (BB-1-3). Having a dynamic pattern in terms of heading direction and overall trajectory clearly helped in finding more accurate beacon location. Table III summarises the simulation results of $B B-1$ series.

\section{3) Beaon pinging interval convergence study}

Every ping contributes to pin pointing the beacon location. More pings received will shorten the time required to find the beacon position. The visualised convergence study is shown in Fig. 10. Only 2 to 4 pings were required to estimate the sufficiently accurate beacon location. Testing conditions were identical to $B B-1-3$ with varying pinging interval frequency which is displayed at the bottom right of each graph.

\section{BB-2: Beacon on the support vessel in the fixed location}

Finally, a dynamic pattern consisted of combination of straight lines and curves was created as shown in Fig. 11 based on the lesson learnt from the previous simulations. In this simulation, combined two error sources were assumed to exist. The red line indicates the estimated path based on DVL data, while the blue and green line was off-set due to navigation error and ice induced error respectively. The first objective of this simulation was to investigate how much error by two error sources would be generated with the second reference (beacon) located outside the IFR. The second object was to evaluate how much the error could be mitigated after the correction method was applied. Simulation was repeated for 1000 runs with random beacon placement. Various beacon pinging intervals used: once per 60, 120, 240, 480 and 1000 seconds.

The total distance error of the final AUV location initially defined by DVL remained $145.1 \mathrm{~m}$ as the AUV operating pattern and ice conditions remained the same. Applying the correction method, the magnitude of the error (DVL error) could be reduced by the difference (Beacon error) between the beacon's true position and the estimated position using Bayesian filtering. Both magnitude and direction of the beacon

Table III. $B B$ - 1 SIMULATIONS PARTICULARS OF THREE PATTERNS.

\begin{tabular}{|c|c|c|c|}
\hline Particulars & BB-1-1 & BB-1-2 & BB-1-3 \\
\hline Pattern & Straight line & Lawn mower & Spiral \\
\hline Vehicle velocity $(\mathrm{m} / \mathrm{s})$ & \multicolumn{3}{|c|}{1.0} \\
\hline Ice velocity $(\mathrm{m} / \mathrm{s})$ & \multicolumn{3}{|c|}{0.08 (to East), 0.08 (to North) } \\
\hline Ice angular velocity $(\mathbf{o} / \mathrm{h})$ & \multicolumn{3}{|c|}{8000} \\
\hline Travel distance $(\mathrm{m})$ & 1000 & 3100 & 9.1 \\
\hline Total displacement $(\mathrm{m})$ & 40.6 & 4.2 & 0.29 \\
\hline Total error rate $(\%)$ & 4.6 & 0.14 & \\
\hline
\end{tabular}
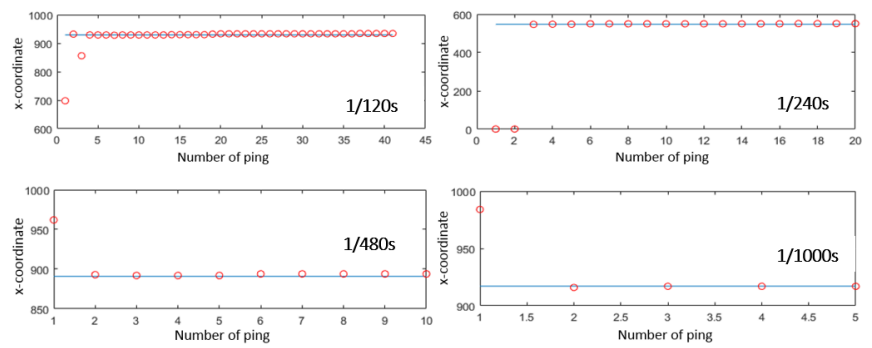

Fig. 10. Pinging interval convergence study with spiral shape pattern.
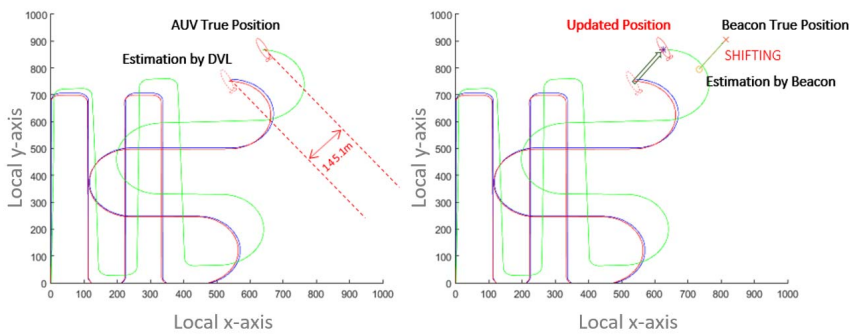

Fig. 11. Correction method application during $B B-2$ simulation. Error between the estimated AUV position by DVL and its true position (left) and the correction method using shifting factor (right).

error should be synchronising to DVL error.

The shift factor reduced the initial error rate of $2.96 \%$ to $1.32 \%$ with frequency of $1 / 60$ second $^{-1}$. The error reduction capability significantly dropped with longer beacon pinging interval frequency. When the interval was 1,000 seconds, the error rate was even higher compared to the initial. Table IV summarise the results of $B B-1$ simulation series.

Through the earlier beacon pinging convergence study of $B B-1-3$, the accuracy rate was found more tolerant to the pinging frequency in the IFR. However, the combined errors due to both DVL and ice-drift made the results more sensitive to pinging frequency. With sufficient acoustic pings, more than half of the initial error was recovered applying the correction method. In Fig. 12, the error reduction of the correction method in terms of magnitude and direction in most of regions

Table IV. SIMULATIONS PARTICULARS OF THREE DIFFERENT PATTERNS

\begin{tabular}{|c|c|c|c|c|c|}
\hline Particular & $\begin{array}{c}B B- \\
2-1\end{array}$ & $\begin{array}{c}B B- \\
2-2\end{array}$ & $\begin{array}{c}B B- \\
2-3\end{array}$ & $\begin{array}{c}B B- \\
2-4\end{array}$ & $\begin{array}{c}B B- \\
2-5\end{array}$ \\
\hline Travel distance $(\mathrm{m})$ & \multicolumn{5}{|c|}{4900} \\
\hline Vehicle velocity $(\mathrm{m} / \mathrm{s})$ & \multicolumn{5}{|c|}{1.0} \\
\hline Ice velocity $(\mathrm{m} / \mathrm{s})$ & \multicolumn{5}{|c|}{0.08 (to East), 0.08 (to North) } \\
\hline Ice rotation $(\% / h)$ & \multicolumn{5}{|c|}{10 (clockwise) } \\
\hline Total displacement (m) & \multicolumn{5}{|c|}{145.1} \\
\hline Beacon frequency $\left(\mathrm{s}^{-1}\right)$ & $1 / 60$ & $1 / 120$ & $1 / 240$ & $1 / 480$ & $1 / 1000$ \\
\hline Reduced displacement (m) & 64.7 & 67.1 & 77.4 & 99.2 & 156.1 \\
\hline Error reduction rate $(\%)$ & 55.4 & 53.7 & 46.7 & 31.6 & -7.6 \\
\hline
\end{tabular}
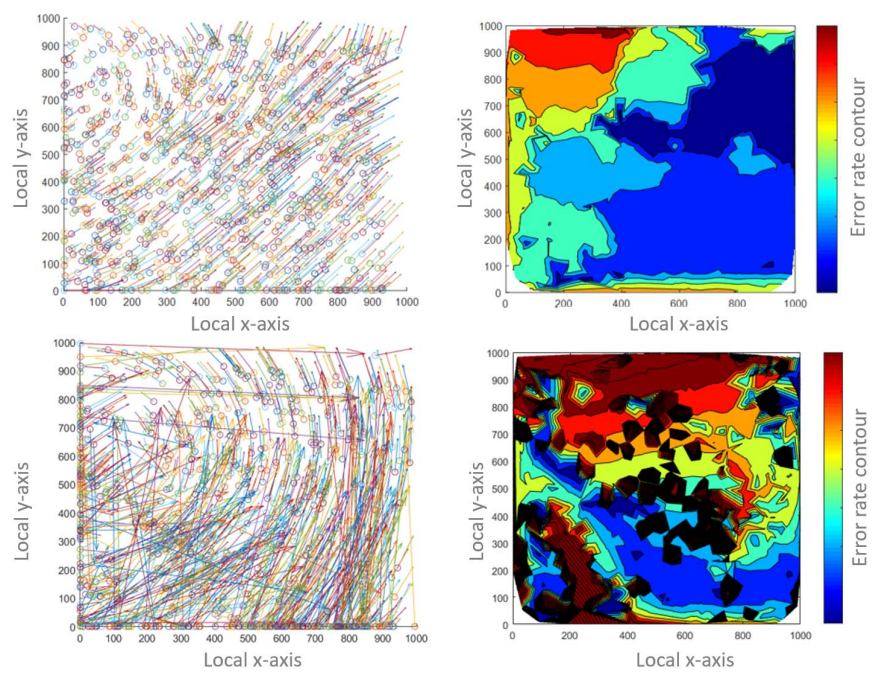

Fig. 12. Vectors of shifting factor while the correction method being applied (Top-left) and the error contour plot (Top-right) of BB-2-1 simulation. Vectors of shifting factor while the correction method being applied (Bottom-left) and the error contour plot (Bottom-right) of $B B-2-5$ simulation. 
appeared to have worked sufficiently except the top left corner on the map. This is more clearly shown in the error contour plot. region in red exhibiting higher error rate compared with other regions. When having excluded the high error region in red, the final DVL error was recovered by $70.8 \%$. Without

Applied correction method with the beacon frequency of $1 / 1000$ seconds $^{-1}$ is visualised in Fig. 12. Without sufficient number of pings, it was not possible to accurately estimate the beacon location regardless of its relative position to AUV trajectory. As shown in the error contour plot, high error occurs across the operating area.

\section{DISCUSSION}

The navigation error in $D D-1$ appeared to linearly distort the AUV trajectory and the resulting offset was relatively small. It, however, accumulated over time, therefore it could possibly cause difficulty to recover the vehicle after a mission involved a long period of time. The error in $D D-2$ exhibited more significant. In practice, freely drifting ice does not always move linearly and also it is not always possible to predict or determine how the ice moved accurately.

In $B B-1$, the error rate remained below $0.3 \%$. The Bayesian filtering successfully localised the beacon after a couple of pings received regardless of the pinging frequency. In $B B-2$, the error reduction was more dependent on the frequency. The more pings received, the more the induced error by ice-drift was compensated. Therefore, the error correcting capability significantly dropped with increasing period of interval time. Another influencing factor on the error rate the bearing angle between the beacon and AUV. Generally, the error rate was reduced by correction method provided the sufficient numbers of acoustic pinging were available.

\section{CONCLUSION AND FUTURE WORK}

This paper presents a few independent sets of algorithms developed to improve the accuracy in AUV localisation results under moving ice utilising Bayesian filter with a pair of an acoustic beacon and a transducer. Major factors contributing to reduction of accuracy in AUV navigation under ice were categorised into two, navigation error and error induced by ice drift. The concept of two reference frames (earth and ice) were introduced. A solution was suggested by having DVL locally referencing the moving ice and a beacon globally referencing the earth. A probabilistic approach in conjunction with the correction method was adopted to reduce the total error rates from the addressed error sources in both frames of reference. The results showed that the averaged error rates were reduced by $70.8 \%$ with the beacon pinging frequency of $1 / 60$ second $^{-1}$ excluding the error concentrated area. In reality, more external error sources exist such as sea currents other than free ice-drift or navigation error. Also, the noise of the acoustic pings can be naturally implemented and further observation of the noise control by the developed algorithms could be investigated.

\section{ACKNOWLEDGMENT}

This research was partly supported by Antarctic Gateway Partnership, A Special Research Initiative of the Australian Research Council.

\section{REFERENCES}

[1] C. Kunz, C. Murphy, H. Singh, C. Pontbriand, R. Sohn, S. Singh, T. Sato, C. Roman, K. Nakamura, M. Jakuba, R. Eustice, R. Camilli and J. Bailey, "Toward extraplanetary under-ice exploration: Robotic steps in the Arctic", Journal of Field Robotics, vol. 26, no. 4, pp. 411-429, 2009.

[2] [2]P. Kimball and S. Rock, "Sonar-based iceberg-relative navigation for autonomous underwater vehicles", Deep Sea Research Part II: Topical Studies in Oceanography, vol. 58, no. 11-12, pp. 1301-1310, 2011.

[3] D. Rudolph and T. Wilson, "Doppler Velocity Log Theory and Preliminary Considerations for Design and Construction", in SoutheastCon, 2012 Proceedings of IEEE, Wyndham Orlando Resort, Orlando, FL, USA, 2012

[4] R. Teledyne, "Instruments Inc. Workhorse navigator Doppler velocity $\log$ (DVL)", Technical report, RD Instruments Inc, Poway, CA, 2011.

[5] P. Kimball and S. Rock, "Mapping of Translating, Rotating Icebergs With an Autonomous Underwater Vehicle", IEEE Journal of Oceanic Engineering, vol. 40, no. 1, pp. 196-208, 2015.

[6] C. Kunz, C. Murphy, R. Camilli, H. Singh, J. Bailey, R. Eustice, M. Jakuba, K. Nakamura, C. Roman and T. Sato, "Deep sea underwater robotic exploration in the ice-covered arctic ocean with AUVs", in International Conference on Intelligent RObots and Systems (IROS 2008), Acropolis Convention Centre, Nice, France, 2008.

[7] A. Kukulya, A. Plueddemann, T. Austin, R. Stokey, M. Purcell, B. Allen, R. Littlfield, L. Freitag, P. Koski and E. Gallimore, "Under-ice operations with a REMUS-100 AUV in the Arctic", in Autonomous Underwater Vehicles (AUV), 2010 IEEE/OES, 2010.

[8] M. Jakuba, C. Roman, H. Singh, C. Murphy, C. Kunz, C. Willis, T. Sato and R. Sohn, "Long-baseline acoustic navigation for under-ice autonomous underwater vehicle operations", Journal of Field Robotics, vol. 25, no. 11-12, pp. 861-879, 2008.

[9] M. Deffenbaugh, H. Schmidt and J. Bellingham, "Acoustic navigation for Arctic under-ice AUV missions", in OCEANS'93. Engineering in Harmony with Ocean. Proceedings, 1993.

[10] J. Keane, A. Forrest, D. Battle, J. Duffy, H. Johannsson and K. Sammut, "Homing for autonomous multi-platform docking", in Pacific International Maritime Conference 2015, sydney Exhibition Centre, Glebe Island, Sydney, 2015.

[11] S. Thrun, W. Burgard and D. Fox, Probabilistic robotics. Cambridge, Mass.: MIT Press, 2010.

[12] T. Bayes, "A Letter from the Late Reverend Mr. Thomas Bayes, F. R. S. to John Canton, M. A. and F. R. S.", Philosophical Transactions of the Royal Society of London, vol. 53, no. 0, pp. 269-271, 1763.

[13] D. Fox, J. Hightower, Lin Liao, D. Schulz and G. Borriello, "Bayesian filtering for location estimation", IEEE Pervasive Computing, vol. 2, no. 3, pp. 24-33, 2003.

[14] D. Fox, "Adapting the Sample Size in Particle Filters Through KLDSampling", The International Journal of Robotics Research, vol. 22, no. 12, pp. 985-1003, 2003. 